\title{
Metamateriales nanoestructurados: avances en el cálculo de sus propiedades ópticas
}

\section{Nanostructured metamaterials: advances in the calculation of their optical properties}

\author{
Ulises R. Meza, ${ }^{*,}$ Bernardo S. Mendoza, ${ }^{*}$ W. L. Mochán**
}

\begin{abstract}
This article aims to present the work that has been done in recent years in the field of nanostructured metamaterials. Mainly, the work done in the Center for Optical Research A. C. in conjunction with the Institute of Physics of the UNAM. These works have focused on the development of new theoretical models that explain the optical properties of nanostructured systems. In addition, these models have been implemented within high-performance computational packages, which has allowed the realization of precise numerical calculations, which allow to know the physical quantities with which a material can be characterized; in this case, nanostructured metamaterials. Mostly, these numerical calculations have been focused on the computation of the dielectric function and non-linear susceptibility, being of great importance to be able to predict its value precisely because they are two fundamental quantities in the understanding of the interaction of light with matter. In addition to this theoretical-numerical development, some results obtained throughout this time are shown, where you can observe the versatility and flexibility of calculations as well as the importance of having such methodologies in material prediction, description and design "measure" of the needs. Finally, the future perspectives of this research are presented, as well as some of the most important challenges in this regard.
\end{abstract}

KEYWORDS: metamaterials, nanostructures, periodic systems, homogenization, effective medium, optical properties, nonlinear optics, permittivity, susceptibility, optical polarization, dichroism, second harmonic generation, nonlinear materials.

RESUMEN: Este artículo tiene como objetivo presentar el trabajo realizado en los últimos años en el campo de metamateriales nanoestructurados. Se muestran principalmente los trabajos realizados en el Centro de Investigaciones en Óptica A. C. en conjunto con el Instituto de Física de la UNAM. Estos trabajos se han centrado en el desarrollo de nuevos modelos teóricos que permitan explicar las propiedades ópticas de sistemás nanoestructurados. Además, dichos modelos se han implementado dentro de paquetes computacionales de alto rendimiento, lo cual ha posibilitado la realización de cálculos numéricos precisos, mismos que permiten conocer las cantidades físicas con las que se puede caracterizar un material; en este caso, metamateriales nanoestructurados. Mayoritariamente, dichos cálculos numéricos se han centrado en el cómputo de la función dieléctrica y la susceptibilidad no lineal, siendo de suma importancia poder predecir su valor de forma precisa por ser dos cantidades fundamentales en el entendimiento de la interacción de la luz con la materia. Además de este desarrollo teórico-numérico, se muestran algunos resultados importantes obtenidos a lo largo de este tiempo, donde se puede

Recibido: 2 de septiembre de 2019.

Aceptado: 16 de octubre de 2019.

*Centro de Investigaciones en Óptica A. C., León, Guanajuato, México.

**Instituto de Ciencias Físicas, Universidad Nacional Autónoma de México, 62251, Cuernavaca, Morelos, México.

•Autor de correspondencia: ulisesrojo@cio.mx 
observar la versatilidad y flexibilidad de los cálculos así como la importancia de contar con dichas metodologías en la predicción, descripción y diseño de materiales "a la medida" de las necesidades. Por último, se presentan las perspectivas a futuro de esta investigación, así como algunos de los retos más importantes al respecto.

PALABRAS CLAVE: metamateriales, nanoestructuras, sistemás periódicos, homogeneización, medio efectivo, propiedades ópticas, óptica no lineal, permitividad, susceptibilidad, polarización óptica, dicroismo, generación de segundo armónico, materiales no lineales.

\section{Introducción}

La definición más usual que se da para un metamaterial es la siguiente: "Un metamaterial es aquel que no existe por sí mismo en la naturaleza y que posee propiedades más allá de las atribuidas a los elementos de los cuales está compuesto. El prefijo "meta" viene del griego que significa "más allá"; la palabra compuesta metamaterial, significa más allá de un material”. Esta es una descripción muy general y suele dar una noción de lo que se está buscando con el desarrollo científico y tecnológico realizado en este campo; nuevos y mejores materiales. En particular, un metamaterial es un material estructurado artificialmente que obtiene sus propiedades de su estructura en lugar de obtenerlas de los materiales constituyentes. Un metamaterial tiene una escala de in-homogeneidad que es mucho más pequeña que la longitud de onda de la luz con la que este material interactúa, y su respuesta electromagnética se expresa en términos de parámetros de material homogeneizado.

A pesar de que desde hace varios siglos ya se realizaban estudios sobre materiales in-homogéneos, no fue sino hasta hace casi 20 años que el término tomó gran relevancia debido a la elaboración de materiales con propiedades ópticas no encontradas en la naturaleza, tales como materiales con índice de refracción negativo y su uso para la fabricación de lentes perfectas (Pendry, 2000), mismás que tienen como antecedentes otros trabajos (Veselago, 1968) y (Smith et al. 2000), donde se predecían los llamados materiales de mano izquierda. Así, en solo unos pocos años, el campo de los metamateriales ha crecido rápidamente, involucrando a investigadores en diversas disciplinas que incluyen física básica, óptica, ciencia de materiales, mecánica e ingeniería. Hoy en día, muchos de estos metamateriales diseñados artificialmente han demostrado tener propiedades electromagnéticas sin precedentes, que no pueden ser obtenidas de forma natural ni mediante técnicas de síntesis de materiales. Las unidades fundamentales que componen la estructura de un metamaterial pueden ser modificadas en tamaño, forma, composición y morfología, lo cual permite que las inclusiones puedan ser diseñadas y colocadas de manera predeterminada para poder lograr el comportamiento deseado. La idea de este tipo de materiales dentro de la comunidad óptica se ha ido adoptando de forma tal que hoy en día es posible encontrar investigadores que se encuentran trabajando en el diseño y fabricación de metamateriales dentro del rango óptico, siendo este el resultado de una combinación de diversas e innovadoras técnicas de nanofabricación, avances en la obtención de imágenes 
a nanoescala, así como el diseño y simulaciones computacionales acerca del comportamiento electromagnético de los mismos. Gracias a estos grandes y rápidos avances, han surgido nuevos campos de investigación, dentro de los que se incluyen metamateriales con gran quiralidad artificial (Plum et al., 2007; Decker et al., 2007), magnetismo óptico (Linden et al., 2004), metamateriales con alta resolución (Liu et al., 2007) e inclusive trabajos de óptica no lineal en metamateriales(Popov et al., 2006; Klein et al., 2007).

Hoy en día, a pesar de que los metamateriales aún no son perfectos, pues usualmente son dispersivos, disipativos y anisotrópicos, son una alternativa en el diseño y fabricación de nuevos materiales. La confirmación de que este tipo de materiales presenta respuestas sin precedentes ha permitido que los científicos se enfoquen en optimizar su diseño, explorar la nueva física que hay detrás de ellos y postular novedosos dispositivos basados en los mismos, trayendo beneficios en distintas áreas como el censado óptico, fabricación de nanoantenas, guías de onda, nanolitografía en nanoescala y circuitos fotónicos, por mencionar algunos.

En las secciones siguientes, se explicará brevemente cómo es que se pueden obtener las propiedades ópticas de un metamaterial y algunas de las teorías más comunes para explicarlos. Además, se realizará una breve exploración dentro de los trabajos teóricos basados en una teoría de homogeneización en específico (Mochán y Barrera, 1985), así como su posterior implementación dentro de un módulo computacional para un uso más eficiente y, por lo tanto, permitir la exploración de un gran número de propiedades ópticas, mismás que en algunos casos son comparadas con las obtenidas por otros métodos, pero que en otros más son de carácter predictivo, debido a lo sólidas que dichas teorías pueden llegar a ser.

\section{Propiedades ópticas en metamateriales}

Los fenómenos electromagnéticos son gobernados en su mayoría por las ecuaciones de Maxwell, las mismás son un conjunto de ecuaciones que relacionan los campos electromagnéticos, sus fuentes y las propiedades de los materiales. La interacción de los campos con la materia modificará el comportamiento de la misma, al poder influir en el acomodo de las cargas eléctricas o inducir polarización en ellas. La manera en que la materia reaccionará ante la presencia de luz, está determinada mediante dos parámetros: la permitividad eléctrica $\epsilon$ y su correspondiente susceptibilidad magnética $\mu$, mismás que están relacionadas con una cantidad de suma importancia dentro de la óptica: el índice de refracción $n=\sqrt{ } \epsilon \mu$.

Un cristal, desde un punto de vista microscópico, está compuesto de un arreglo periódico de átomos ordenados en una manera específica. En cada molécula atómica, pequeños dipolos eléctricos pueden ser excitados por el campo eléctrico correspondiente a una onda de luz incidente y, subsecuentemente, estos dipolos comenzarán a radiar la energía después de un cierto tiempo. A 
pesar de que la distribución del campo eléctrico dentro del cristal es no uniforme, la luz no alcanza a percibir estas in-homogeneidades. Desde la escala macroscópica, las respuestas características de la estructura in-homogénea son promediadas y debido a ello se pueden establecer relaciones entre los campos macroscópicos de las ecuaciones de Maxwell; el campo eléctrico $E$, el campo magnético $H$, el desplazamiento eléctrico $D$ y la densidad de flujo magnético $B$. De estas relaciones se pueden extraer la permitividad y permeabilidad de cada material.

De la misma manera, en un metamaterial se presentan in-homogeneidades debido a la composición del mismo, además de que el tamaño de las mismás es muy pequeño comparado con la longitud de onda $\lambda$ de la luz a la que se desea que funcione el metamaterial que se ha diseñado. El tamaño de estas inclusiones está relacionado con el tamaño de la red artificial que fue construida a partir de la estructuración del material, es decir, el acomodo de estas in-homogeneidades. En este caso pasa lo mismo que en un cristal, es complicado analizar a la luz y sus interacciones entre estos "meta-átomos", pero desde un nivel macroscópico, el material parecerá ser un medio homogéneo para la luz. De esta manera, la respuesta electromagnética en un metamaterial puede ser descrita de manera similar que en los materiales convencionales, mediante la homogeneización de sus propiedades microscópicas que resultarán en parámetros efectivos $\left(\epsilon_{e f f}, \mu_{e f f}\right)$. Por lo tanto, un sistema nanoestructurado puede presentar propiedades ópticas distintas a las de un material convencional, es por ello que la predicción de sus propiedades se ha vuelto de gran importancia y cada vez se requieren métodos más eficientes para realizar los cálculos de dichas propiedades.

En este sentido, existen varios enfoques sobre cómo calcular las propiedades de metamateriales, específicamente de sistemás nanoestructurados. Lo que se hace típicamente es un proceso de homogeneización entre las propiedades de sus componentes, dando como resultado una propiedad efectiva que es la que describe el comportamiento de la luz ante estas estructuras (McPhedran y Milton, 1981; Mochán y Barrera, 1985). Los tamaños de estas estructuras pueden variar dependiendo de la aplicación que se les quiera dar. Dentro de las propiedades ópticas, un parámetro de referencia para el diseño de estos materiales es la longitud de onda de la luz con las que deseamos que el metamaterial funcione. El tamaño de las estructuras que se pueden fabricar, en comparación con la longitud de onda de la luz, suele ser alrededor de 5 veces menos, lo cual permite que la luz al interactuar vea un material promedio y se obtengan propiedades diferentes a las de sus componentes, en algunos casos propiedades que no son encontradas en la naturaleza.

Con los avances de las técnicas de fabricación, así como del poder de cómputo que se han tenido en los últimos años, se espera poder realizar una exploración amplia de los parámetros que caracterizan un metamaterial y que hace posible que presenten propiedades electromagnéticas interesantes, por esto se piensa que dentro de poco las limitantes para tener estos materiales serán muy reducidas, tanto desde el punto de vista teórico como desde 
el experimental, teniendo así en nuestras manos un sin fin de posibilidades para poder manipular la luz a nuestra conveniencia.

\section{Respuesta óptica efectiva de metamateriales nanoestructurados}

Como ya se mencionó en la sección anterior, la respuesta macroscópica efectiva de un metamaterial nanoestructurado puede ser obtenida mediante la homogeneización de las propiedades microscópicas de sus componentes. Existen varios modelos teóricos empleados para describir cómo es el comportamiento de la luz ante sistemás no homogéneos, algunos de los más utilizados son los modelos de Maxwell Garnet (MG), mismo que supone un material que funciona como matriz con inclusiones esféricas dentro de él. En este modelo, es importante tomar en cuenta qué función tiene cada uno de los materiales, pues a medida que la fracción de llenado aumenta, ya no es tan fácil distinguir entre el material matriz y la inclusión. Existe otro modelo basado en la fórmula de Brugeman (FB), muy similar a MG, con la característica de que en esta aproximación, la forma de las inclusiones no debe ser forzosamente esférica, estas pueden tomar diferentes formás, incluso pueden ser de dos o más materiales, caracterizados por sus correspondientes funciones dieléctricas. ${ }^{1}$ Aunque estos son dos de los modelos más utilizados para poder describir el comportamiento de materiales de este tipo, muchos autores han contribuido en el desarrollo de nuevos modelos teóricos que permitan predecir dichas propiedades, de suerte tal que se puedan mejorar algunas de las limitantes que se tienen en cada método. Además de estos, se ha desarrollado una teoría para describir la respuesta en una representación específica que resulta en una poderosa herramienta para calcular la respuesta dieléctrica macroscópica (Mochán y Barrera, 1985). En esta teoría, la respuesta del sistema es obtenida a partir de las ecuaciones constitutivas microscópicas debido a la eliminación de las fluctuaciones de los campos, usando las ecuaciones de Maxwell y resolviéndolas para el desplazamiento eléctrico macroscópico en términos del correspondiente campo eléctrico. Además del promedio de la función dieléctrica, este formalismo incorpora los efectos de la rápida variación de las componentes de Fourier de la respuesta microscópica y la influencia que estas tienen sobre la respuesta macroscópica. Algunos procedimientos de homogeneización, similares al anteriormente descrito, han sido propuestos por algunos otros autores (Krokhin et al., 2002; Datta et al.,1993). Adicional a esto, pueden realizarse algunas consideraciones en este método o restringirse a ciertas condiciones con el fin de poder obtener estas respuestas en términos solamente de la forma geométrica de las inclusiones, de su arreglo periódico y de las funciones dieléctricas de sus componentes para periodos más pequeños que la longitud de onda de la luz in-

1 Para una breve introducción a estas aproximaciones, véase Markel (2016). 
cidente. Con el fin de tener un modelo que pueda ser utilizado para hacer cálculos numéricos de estas cantidades de una manera sencilla, se propone una nueva forma de conocer estas propiedades (Ortiz et al., 2009), donde se reproducen resultados previamente reportados para sistemás bien conocidos (Tao et al., 1990; Bergman y Dunn, 1992; Milton, 1981). También se realizan las correspondientes pruebas de convergencia mediante la revisión del teorema de Keller y el cálculo de los respectivos coeficientes de Keller (Keller, 1963 y 1964; Nevard y Keller, 1985). Una vez que se dio validez a este nuevo modelo para calcular las propiedades macroscópicas de un metamaterial, se aplicó a sistemás reportados anteriormente, es el caso de un sistema 2D cuadrado de prismás rectangulares infinitos con sus diagonales alineadas con los lados de la celda unitaria cuadrada que se muestra en la figura 1.

El sistema había sido propuesto anteriormente por (Milton et al., 1981). Los parámetros escogidos para estos cálculos, fue una función dieléctrica de $\epsilon p=5.0$, correspondiente a una inclusión de un dieléctrico en el vacío, y una fracción de llenado $f=0.3$. Se muestran en la figura $2 a$, las comparaciones con la formulación de MG y con los cálculos hechos anteriormente, mostrando la discrepancia que existe entre ellos. Las pruebas de convergencia se muestran en la figura $2 \mathrm{~b}$ y se observa que el método converge de mejor manera para tamaños de celda considerablemente más pequeños que la longitud de onda de la luz incidente. El promedio de la convergencia de este método es comparable con la presentada anteriormente (Sözüer et al., 1992).

La validación de estos resultados en comparación con los presentados anteriormente con otros métodos, permite la exploración de más sistemás, es decir, modificando parámetros como lo son las dimensiones de la celda unitaria, el tamaño de los prismás rectangulares, cambiar el dieléctrico del que están hechas estas inclusiones e incluso variar su fracción de llenado, tal

FIGURA 1. Representación esquemática de un metamaterial compuesto por dos materiales caracterizados por $\epsilon_{h}$ correspondiente al host y $\epsilon_{p}$ a la inclusión. Además $L_{x}$ y $L_{y}$ corresponden al tamaño de la celda unitaria en las direcciones $x$ y $y$, respectivamente.

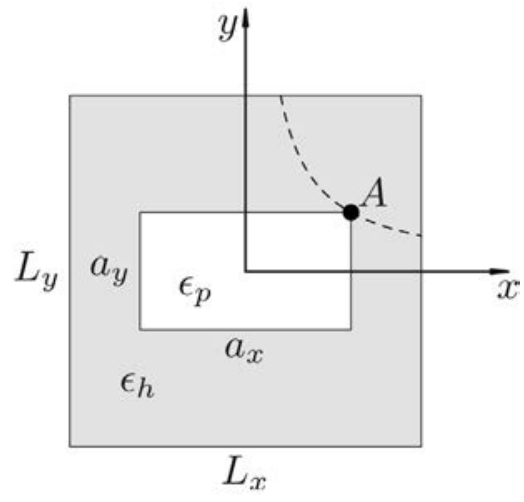

Fuente: Tomada de Ortiz et al. (2009). 
FIGURA 2. En a) se muestran los cálculos realizados de la función dieléctrica macroscópica para el sistema presentado en la figura 1. Estos resultados se comparan entre los presentados en trabajos anteriores Tao et al.(1990); Bergman y Dunn (1992); Milton (1981) y el presentado en Ortiz et al. (2009). En b), se muestran los coeficientes de Keller calculados para el mismo sistema, como prueba de convergencia del cálculo, para distintos tamaños de la celda en comparación con la longitud de onda.

a)

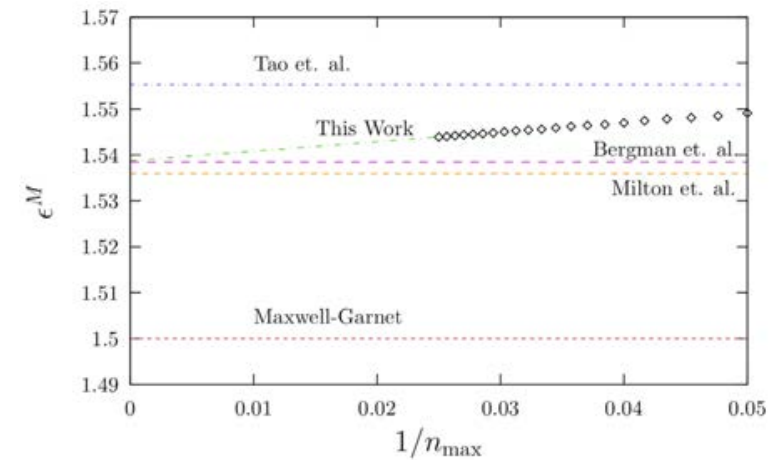

Fuente: Tomada y adaptada de Ortiz et al. (2009). b)

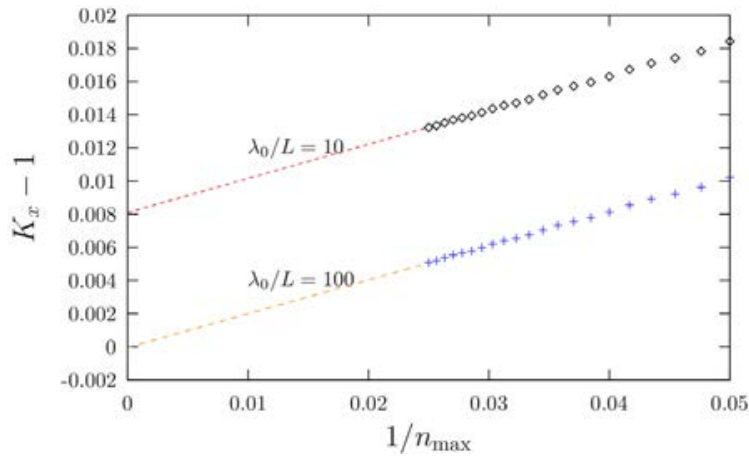

como se muestra en la figura 3, donde se muestra la variación de la función dieléctrica macroscópica en función de la fracción de llenado de las inclusiones. Este sistema corresponde al mismo utilizado para los resultados de la figura $2 \mathrm{a}$, simplemente variando las dimensiones de la inclusión y por otra parte, comparando contra los métodos ya citados.

Por otra parte, es posible explicar el comportamiento de estos sistemás nanoestructurados en términos de propiedades ópticas como lo son la transmisión y la reflexión. Es posible por la flexibilidad de estos cálculos, hacer un estudio sobre un rango amplio de frecuencias, analizando así su comportamiento en distintas zonas del espectro electromagnético. Aquí se presenta un hecho importante, pues dependiendo de la polarización de la luz con la

FIGURA 3. Se muestra el cálculo de la función dieléctrica macroscópica como función de su fracción de llenado.

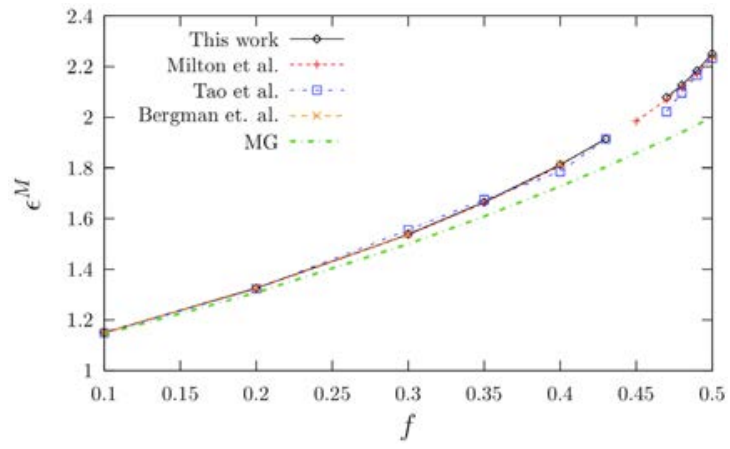

Fuente: Tomada de Ortiz et al. 2009. 
FIGURA 4. Cambios en la reflectancia del oro al modificarlo con inclusiones como se muestra en el esquema de la figura 1, con $\epsilon_{p}=4 \mathrm{y}$ variando las dimensiones de las mismas a lo largo de la dirección $\mathrm{x}$.

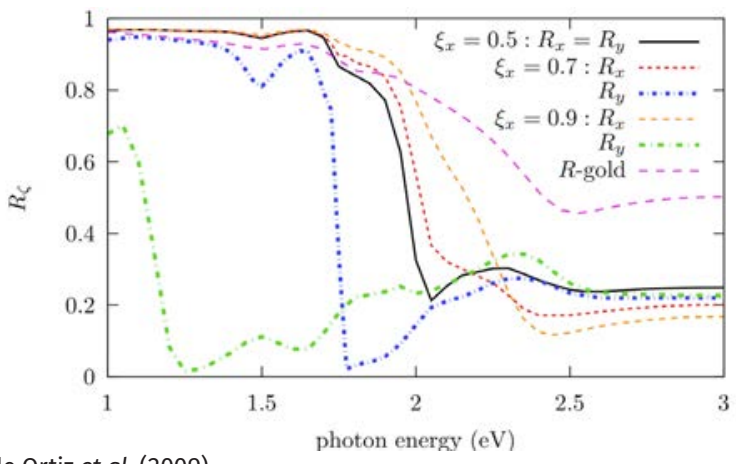

Fuente: Tomada de Ortiz et al. (2009).

que se esté incidiendo en el metamaterial, este podrá mostrar anisotropía en ciertas direcciones. Este hecho se muestra en la figura 4, donde el sistema utilizado fue una película de oro que funciona como el anfitrión e inclusiones de un dieléctrico con $\epsilon p=4$, fijando las dimensiones del lado correspondiente al eje " $y$ ", haciendo variaciones en el lado correspondiente al eje " $x$ ", se puede observar un comportamiento inusual en el oro. El oro presenta una gran reflectancia en el rango entre $1 \mathrm{eV}$ y $2 \mathrm{eV}$, pero al hacer este tipo de modificaciones con ayuda de estas nanoestructuras, podemos observar un decrecimiento en la reflectancia. De la misma manera, se pueden realizar nanoestructuras con distintas geometrías, tal es el caso de una matriz de plata a la cual se le hacen inclusiones en forma de cilindros con una función dieléctrica de $\epsilon p=4$, estos pueden ir modificando su radio y con ello la fracción de llenado, presentando nuevamente anisotropía y una modificación en su reflectancia como función de la fracción de llenado del mismo.

Como se puede observar, el desarrollo de este método, ha permitido realizar cálculos de la respuesta macroscópica en un metamaterial de una manera alternativa a las ya conocidas, con una gran coincidencia con otros métodos bajo ciertas consideraciones. El uso de un gran número de ondas planas es requerido para tener convergencia en los cálculos.

\section{Proceso de homogeneización eficiente para el cálculo de propiedades ópticas en metamateriales.}

De la misma manera en que existen distintas formás de realizar el proceso de homogeneización de las propiedades en un sistema nanoestructurado, existen diversas formás en que estas sean implementadas computacionalmente. El hecho de contar con métodos cada vez más eficientes para el cómputo de estas propiedades, permite una basta exploración de diversas configuraciones en sistemás nanoestructurados. Dichas configuraciones pueden ser pensadas 
como modificaciones en la geometría de la celda, la periodicidad, la forma de las inclusiones o el cambio de las propiedades de los materiales de los que se compondrá la estructura. En este sentido, cabe mencionar que a pesar de que los metamateriales más comunes se basan en una combinación "metal-dieléctrico", no existe ninguna restricción al respecto sobre qué materiales pueden ser utilizados para la elaboración de los mismos, esto dependerá de las características que se deseen tener en el nuevo material. De esta forma, la exploración de distintos sistemás es deseada para poder tener una explicación completa de lo que pasa al modificar un parámetro entre los disponibles. Así pues, el análisis hecho a partir de predicciones teóricas es de gran ayuda en las etapas de diseño de metamateriales. Para hacer este proceso aún más eficiente, es deseable que los algoritmos o métodos que son utilizados computacionalmente para implementar los modelos teóricos que se han mencionado, sean lo más rápidos y precisos posibles. Tal es el caso del método de recursión de Haydock. Si bien este método fue desarrollado con la intención de resolver la ecuación de Schrödinger en el área de la mecánica cuántica (Haydock,1980), el identificar una analogía entre las ecuaciones ahí descritas y la forma en que se solucionan respecto al método para homogeneizar las propiedades en un metamaterial presentadas en Ortiz et al. (2009), ha permitido una implementación de este método numérico eficiente, el cual reduce en varios órdenes de magnitud los tiempos de cálculo de las propiedades, permitiendo con ello una exploración más extensa que al utilizar los métodos tradicionales para la solución de las ecuaciones que gobiernan estos fenómenos.

La teoría utilizada aquí, proviene del mismo modelo de homogeneización propuesto por Mochán y Barrera (1985), pero cambiando significativamente la forma en que este se implementara computacionalmente. A grandes rasgos, podemos hablar de una manera más eficiente de invertir una matriz, paso necesario para el cálculo de la función dieléctrica macroscópica. Este proceso eficiente es posible mediante el cambio de base de la función característica que describe la geometría y las funciones dieléctricas de los componentes del metamaterial. Al representar estos elementos de matriz en dicha base, es posible representarlos dentro de una matriz tridiagonal, lo cual tiene como consecuencia que su inversión sea trivial mediante una fracción continuada. Para hacer este proceso, basta simplemente con calcular los coeficientes de Haydock para tener la representación correcta de la función característica de nuestro metamaterial bajo estudio (Mochán et al., 2010; Cortes et al., 2010).

Para esta nueva implementación computacional, se han realizado las mismás pruebas contra los métodos conocidos, así como contra los resultados reportados anteriormente. Como se puede observar en la figura 5, los resultados obtenidos para la respuesta macroscópica, como función de su fracción de llenado, están en gran concordancia con los predichos por MG y FB, para arreglos simples cúbicos con inclusiones esféricas con $\epsilon_{b}=4$ incrustadas en un medio con $\epsilon_{a}=1$. Estos cálculos fueron realizados tanto para un sistema 3D, descrito anteriormente (figura 6a), como para un sistema 2D (fi- 
Mundo Nano | ARTículos DE REVISión | www.mundonano.unam.mx

13(24), 1e-18e, enero-junio 2020 | http://dx.doi.org/10.22201/ceiich.24485691e.2020.25.69611

Ulises R. Meza, Bernardo S. Mendoza, W. L. Mochán

FIGURA 5. Comparación de los cálculos realizados para la función dieléctrica mascroscópica: a) para un sistema 3D con inclusiones esféricas y, b) un sistema 2D con inclusiones cuadradas.

a)

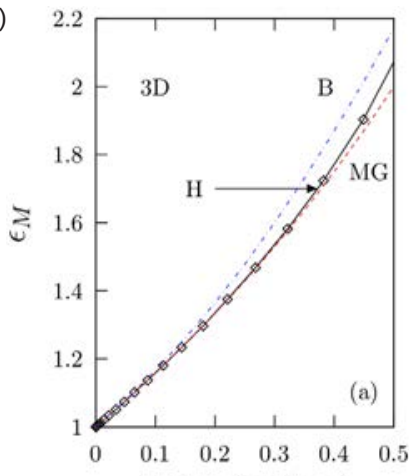

Filling fraction $f$

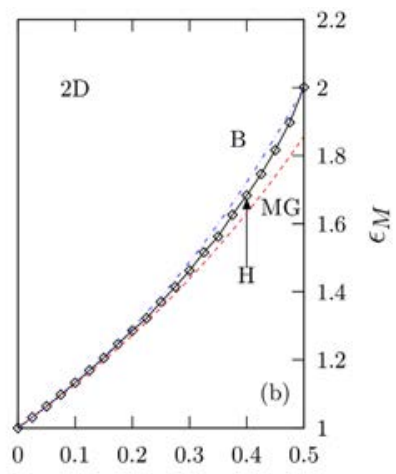

Filling fraction $f$

b)

Nota: Las letras en las gráficas corresponden a los métodos utilizados para los cáculos, donde (MG) corresponde a Maxwell-Garnet, (H) al método de Haydock y, (B) a la fórmula de Brugeman. Fuente: Tomada de Mochán et al. (2010).

FIGURA 6. Película de Au de $200 \mathrm{~nm}$, con inclusiones dieléctricas: a) en forma de esferas y, b) cilindros. En ambas figuras se muestra la transmitancia en función de la energía de la luz incidente.

a)

$$
\lambda_{0}(\mathrm{~nm}) \quad \lambda_{0}(\mathrm{~nm})
$$

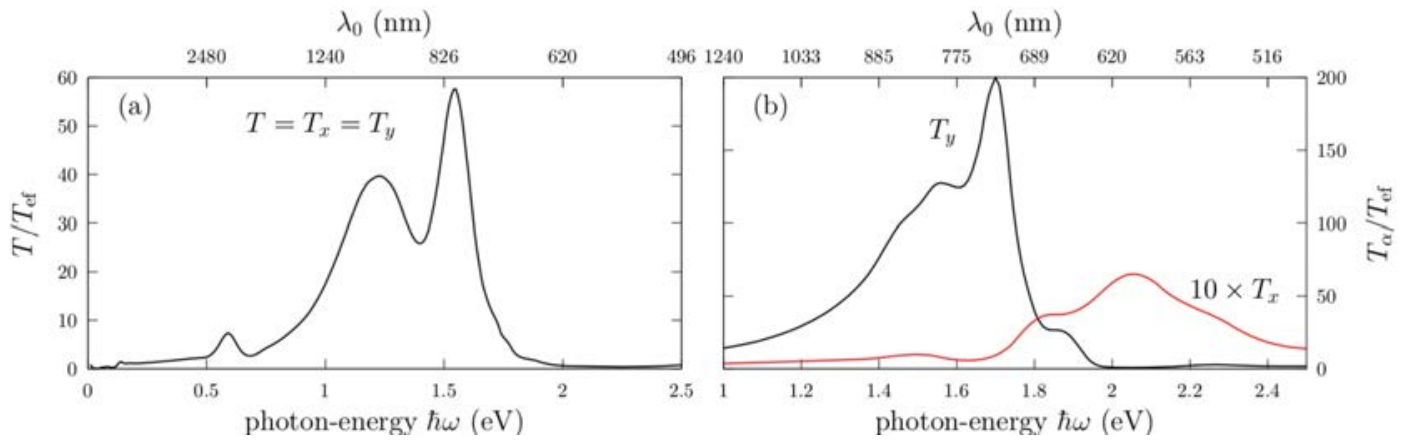

Fuente: Tomada de Mochán et al. (2010).

gura $6 \mathrm{~b}$ ) basado en inclusiones de forma cuadrada, con los mismos valores de su función dieléctrica.

La implementación de esta nueva forma de hacer los cálculos, basados en el método de recursión de Haydock, reduce considerablemente el tiempo de cálculo. Cabe mencionar que para los cálculos realizados con este método, se utilizó la aproximación de longitud de onda larga lo cual impone una restricción en el tamaño de la celda unitaria a. Esta restricción implica que el tamaño de la celda unitaria de nuestro sistema sea del orden de $a \leq \lambda_{0} / 5$. Si ponemos atención en los resultados mostrados en la figura 6 , donde se muestra la transmitancia para una película de oro de $200 \mathrm{~nm}$ con inclusiones de un 
dieléctrico con $\epsilon_{b}=4$ y geometrías esféricas y cilíndricas, respectivamente, podemos observar que las regiones de interés están entre $1 \mathrm{eV}$ y $2 \mathrm{eV}$, por lo que el tamaño de $a$ será aproximadamente de $100 \mathrm{~nm}$, dimensiones para las cuales la fabricación de dichos materiales no supone un gran reto.

\section{Metamateriales nanoestructurados birrefringentes y polarización óptica a la medida}

Contando con un método eficiente para conocer las respuestas macroscópicas en un metamaterial como lo es la función dieléctrica, es posible calcular algunas otras propiedades y analizar otro tipo de fenómenos en estos materiales, tal es el caso de la birrefringencia, y, por otra parte, la posibilidad de polarizar la luz al interactuar esta con determinadas estructuras. En este punto, es importante resaltar que no necesariamente se tienen que encontrar propiedades exóticas en metamateriales, algunas veces basta con una mejora sustancial en la respuesta que estos presentan en comparación con los materiales encontrados de forma natural. Esto puede ser muy claro si observamos el comportamiento óptico anisotrópico que se puede inducir al nanoestructurar un material. Esta condición puede generar distintos comportamientos dependiendo de la orientación de las inclusiones. Tal como se muestra en la figura 7, con una estructura de este estilo podemos variar los ejes cristalinos en nuestro material cambiando de esta manera la forma en que la luz incidente va a interactuar con él.

Este sistema periódico es creado a partir de un dieléctrico con $\epsilon_{a}=4$ e inclusiones de oro con $\epsilon_{b}=\epsilon_{A u}$, misma que es compleja. Al hacer variaciones en la fracción de llenado de las inclusiones y, por otra parte, cambiar su orientación, podemos encontrar que las fases conductoras en el metamaterial tendrán distintas

FIGURA 7. Inclusiones en forma de elipse donde se muestra la rotación de sus semiejes, en este caso el semieje mayor es rotado un ángulo $\theta$, respecto al plano xy del metamaterial. Nótese que el semieje mayor no necesariamente coincide con el eje y del material, debido a las interacciones con las inclusiones vecinas.

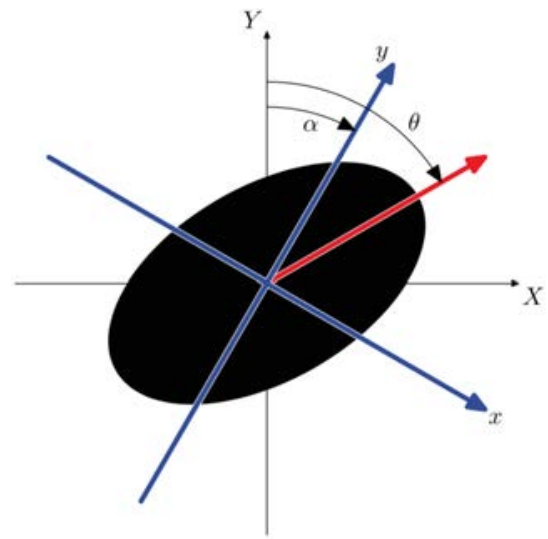

Fuente: Tomada de Mendoza y Mochán (2012). 
FIGURA 8. Reflectividad del oro en comparación con un sistema nanoestructurado con inclusiones elípticas con $\epsilon_{\mathrm{a}}=4$. A diferentes ángulos de rotación $\theta$ del eje mayor de las elipses, la reflectividad muestra distintos comportamientos.

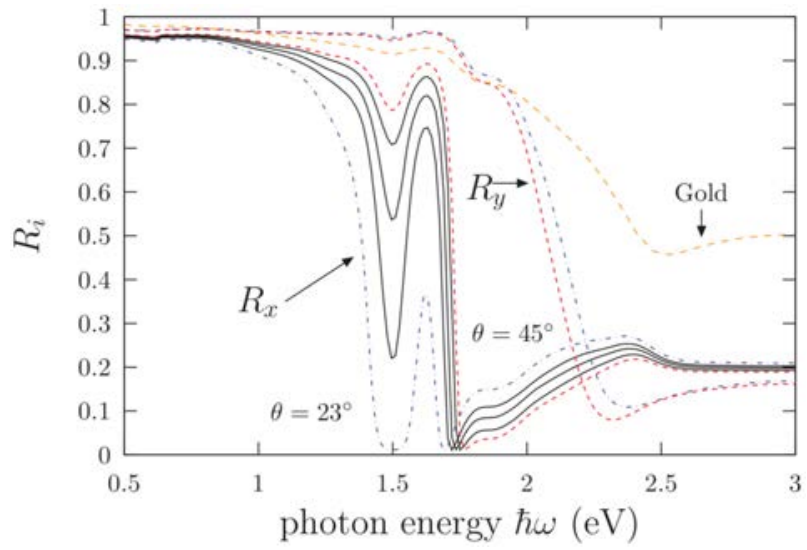

Fuente: Tomada de Mendoza y Mochán (2012).

configuraciones. Así pues, para una fase conductora continua, el comportamiento del metamaterial a bajas frecuencias será cuasimetálico, mientras que para una fase conductora que presenta cuellos de botella, el comportamiento a altas frecuencias será más bien parecido a un comportamiento dieléctrico. El cambio en las fases conductoras, se puede dar por el simple hecho de cambiar la polarización de la luz que esta incidiendo sobre el metamaterial, teniendo así la posibilidad de hacer un barrido en un amplio rango del espectro para una misma estructura y analizar los comportamientos anómalos que estos puedan presentar.

Por otra parte, es conocido que algunos materiales tienen la capacidad de modificar el estado de polarización de una onda de luz que se está propagando, esto es debido a la actividad óptica que se puede presentar en ellos. Es posible utilizar los mismos conceptos para analizar cómo es que un metamaterial puede modificar los estados de polarización de la luz, pero esta vez a una escala nanométrica. Es posible diseñar cierto tipo de polarizadores a partir de nanoestructuras muy específicas de oro y plata las cuales modificarán no solo la cantidad de luz que es reflejada y transmitida (si se considera una película delgada), sino que a su vez se puede generar luz elípticamente polarizada a partir de luz linealmente polarizada o no polarizada (figura 9).

Como ya se mencionó anteriormente, de la misma manera que es posible generar luz polarizada, también existe la posibilidad de generar dicroismo circular a través de ciertos arreglos periódicos. Tal como se muestra en la figura 10, el dicroismo circular generado por un metamaterial hecho con una película de plata depositada en un sustrato de vidrio con inclusiones rectangulares de aire. El desplazamiento entre cada uno de los agujeros rectangulares se ha optimizado mediante rutinas de optimización basadas en MINUIT, con lo cual se pudo obtener dicha respuesta. 
FIGURA 9. En a) se muestra la geometría de un metamaterial hecho a base de agujeros en forma de cruz en una película de un conductor. En b), se muestra la reflectancia y la transmitancia para un sistema con la geometría mostrada en a), hecho con una película de plata sobre un sustrato de vidrio con arreglos periódicos de agujeros en forma de cruz. $L_{x}=0.963 a, L_{y}=a, W_{x}=0.249 a$ y $W_{y}=0.324 a$, aquí las elipses muestran cómo va cambiando el estado de polarización de la luz cuando esta incide con un ángulo de $\theta=45^{\circ}$.

a)

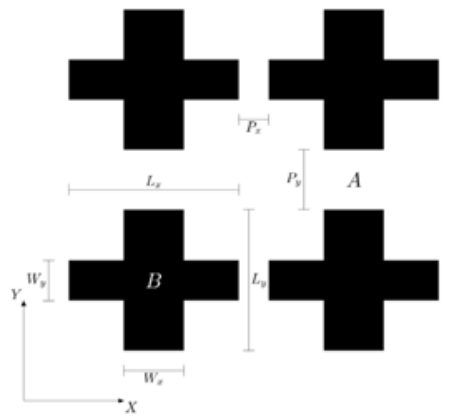

b)

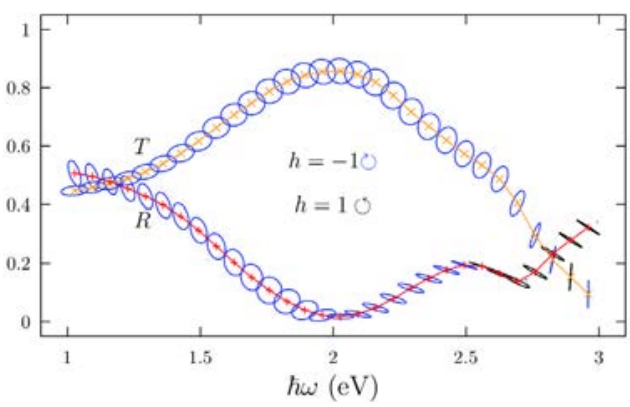

Fuente: Tomada y adaptada de Mendoza y Mochán (2016).

FIGURA 10. Estructura basada en agujeros rectangulares en una película de plata a), la optimización de sus parámetros geométricos permite observar un alto dicroismo circular b).

a)

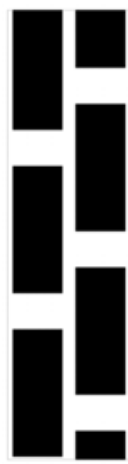

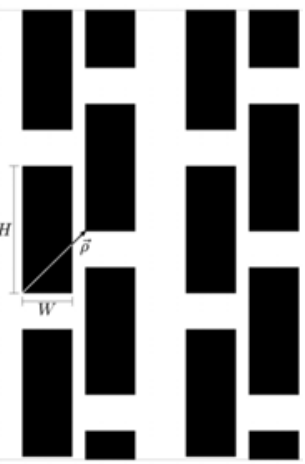

b)

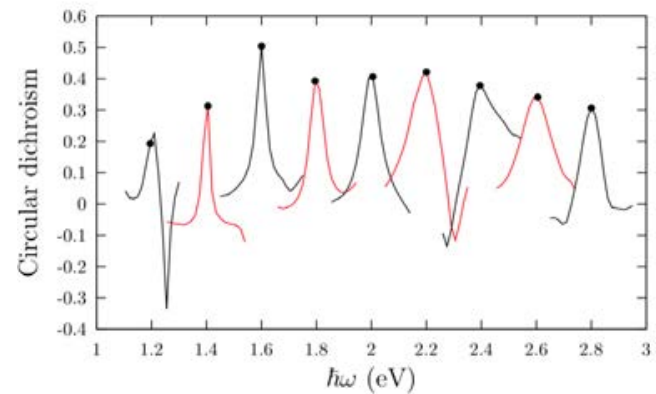

Fuente: Tomada y adaptada de Mendoza y Mochán (2016).

\section{Óptica no lineal en metamateriales nanoestructurados}

La óptica no lineal es una rama con numerosas aplicaciones científicas y tecnológicas de gran importancia desde sus inicios y su desarrollo ha sido posible, en gran medida, gracias a la invención del láser. Dentro de la amplia gamma de fenómenos ópticos no lineales que existen, tales como generación de suma de frecuencias (GSF), generación de diferencia de frecuencias (GDF), mezclado de cuatro ondas (MCO), amplificación óptica paramétrica (AOP) y quizá el más conocido y con un gran número de aplicaciones sea la generación de segundo armónico (GSA). Todos estos fenómenos pueden ser descri- 
tos mediante una cantidad física denominada susceptibilidad no lineal, la cual nos dice qué tan "susceptible" es el material para generar fenómenos no lineales. Dicha propiedad puede ser de distintos órdenes, siendo la susceptibilidad de segundo orden la responsable del fenómeno de GSA. Desde un punto de vista microscópico, los sistemás que permiten generar este fenómeno deben cumplir ciertas condiciones, siendo fundamental la no centrosimetría; los procesos no lineales de segundo orden están prohibidos en materiales que presentan centro-simetría (Schaich y Liebsch, 1988).

Contando con los métodos adecuados para calcular las propiedades microscópicas en metamateriales, es posible también analizar la parte no lineal de los mismos. Típicamente, se ha buscado generar fenómenos no lineales en materiales que por sí mismos no son capaces de hacerlo, basándose simplemente en la modificación de su estructura a un nivel macroscópico (Brudny et al., 2003; Rodrigo et al., 2015; Krasnok et al., 2018). En este sentido, podemos mencionar nanoestructuración de películas de plata. La plata cuenta con una estructura interna que es centro-simétrica y la GSA no está permitida. Con una idea muy sencilla, la cual consiste en perforar dichas películas, es posible cambiar la estructura, pero solo hasta un nivel nanométrico. En este caso, la plata funcionará como el material anfitrión y las perforaciones con una determinada geometría serán las inclusiones del metamaterial. No obstante, estructurar aleatoriamente el material no es suficiente para generar procesos no lineales, se necesita escoger con precisión la manera de hacerlo para que pueda ser posible inducir un cambio en su geometría y así generar no centro-simetría en el material, de esta manera, la luz podrá percibir una estructura no centrosimétrica y así ser capaz de generar segundo armónico (Mochán et al., 2014). Tal como se muestra en la figura 11a, las propiedades no lineales de un metamaterial son descritas mediante su tensor de susceptibilidad no lineal $\chi^{(2)}$, el cual presentará cambios significativos conforme la estructura en el metamaterial esta cambiando. La inducción de no centro-simetría en el metamaterial generará que las resonancias en $\chi^{(2)}$ vayan en aumento, e, incluso, con el cambio en los parámetros geométricos de las inclusiones, permiten una sintonización de las mismás a diferentes frecuencias. Dado que esta propiedad está relacionada con la polarización de segundo orden $P^{(2)}$, se puede observar cómo es que la alineación de los dipolos en el metamaterial coincide para las polarizaciones (de la luz incidente) necesarias para generar las componentes de la susceptibilidad mostradas anteriormente. Es debido a esto que aparecen tales máximos, es decir, el metamaterial es altamente polarizable a esa frecuencia y es por ello que existe una respuesta no lineal, en este caso de GSA. Como se muestra en la figura 11b, la intensidad de los mapas de polarización coincide con las componentes más intensas la susceptibilidad no lineal, estos mapas fueron calculados precisamente en la energía donde se presentan los máximos en $\chi^{(2)}$.

Por último, es posible hacer un análisis a partir de la distribución del campo eléctrico longitudinal y la densidad de carga superficial para ciertas configuraciones de la estructura y a determinadas energías. Como se puede 
FIGURA 11. En a) se muestra la susceptibilidad no lineal de segundo orden $\chi^{(2)}$ para un metamaterial nanoestructurado hecho con una placa de plata e inclusiones de vacío en forma de cruz, para la misma estructura se presenta la polarización no lineal correspondiente b) y, por último, sus correspondientes campos eléctricos y la densidad de carga en c), en las frecuencias de interés.

a)
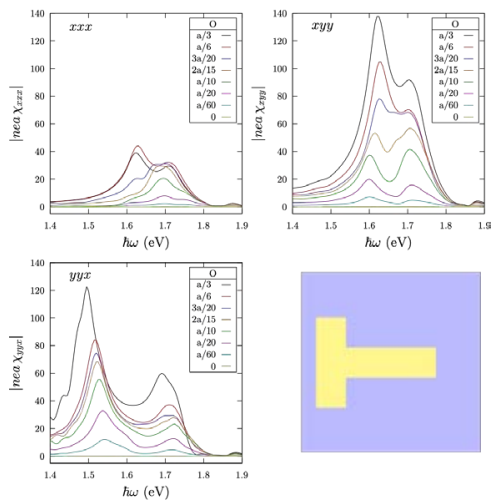

b)

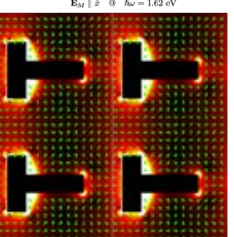

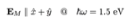

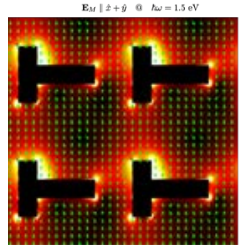

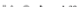

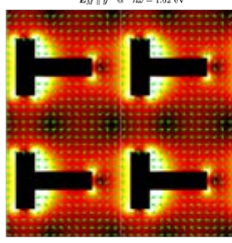

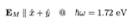

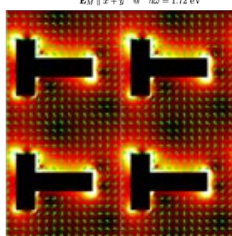

c)

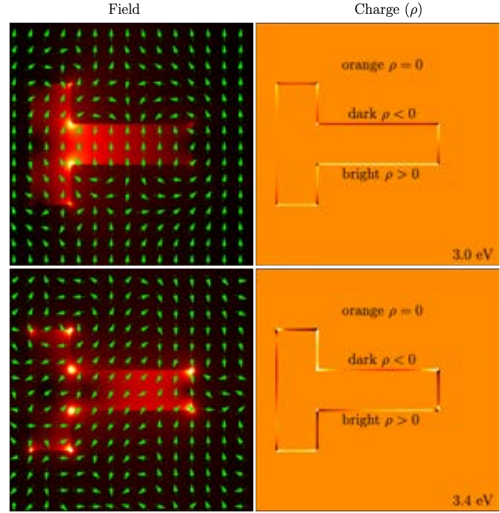

Fuente: Tomada y adaptada de Meza et al. (2019).

apreciar, la carga se distribuye en la interface entre las inclusiones y el material anfitrión, y el campo eléctrico parece que induce ciertos modos dipolares y cuadrupolares, responsables de GSA.

\section{Conclusiones}

El desarrollo de metamateriales nanoestructurados depende en gran medida de las capacidades tecnológicas que se tengan para poder fabricarlos, pero también de las teorías y métodos computacionales disponibles que permitan hacer una predicción precisa y óptima para así ayudar a un diseño eficiente de los mismos. Esto abre la posibilidad de poder experimentar de una manera rápida con estos sistemás sin la necesidad de tener que fabricarlos y analizarlos con la incertidumbre que esto conlleva. Como se ha mencionado, el desarrollo de estos materiales es importante pues tienen usos diversos: pueden ser aplicados en áreas muy distintas; ya sea en telecomunicaciones, como en biosensores o incluso en el desarrollo de nuevas fuentes de luz, entre muchos otros.

Los nuevos fenómenos, que pueden ser predichos por algunos modelos teóricos, así como la mejora en las respuesta ópticas de ciertos materiales, tienen una gran relevancia hoy en día, ya que al poder ser capaces de llevar estos nuevos materiales a una escala nanométrica, estamos siendo capaces de manipular la luz a niveles nunca antes imaginados, y como consecuencia de esto permite un desarrollo científico y tecnológico cada vez más importante.

Así como es fundamental el desarrollo tecnológico para su fabricación, el desarrollo de nuevos métodos computacionales, basados en teorías só- 
lidas que describen el comportamiento de metamateriales, también lo es, pues estos se vuelven herramientas indispensables para la investigación en este campo. En primer lugar permite dar una descripción adecuada de los fenómenos que se están observando pero, por otro lado, contribuye a la optimización de recursos de distintos tipos. Si bien aún se tienen algunas restricciones y complicaciones para la fabricación de estos materiales, la mejora continua en ciencia y tecnología está permitiendo que actualmente se estén creando en cierta medida, los materiales del futuro.

\section{Referencias}

Bergman y Dunn. (1992). Bulk effective dielectric constant of a composite with a periodic microgeometry. Physical Review. B, Condensed Matter, 45(23): 1326271, junio 15. http://dx.doi.org/10.1103/physrevb.45.13262

Brudny, V. L., Mochán, W. L., Maytorena. J. A. Mendoza, B. S. (2003). Second harmonic generation from a collection of nanoparticles. Physica Status Solidi (b), 240(3): 518-26, diciembre 1. http://dx.doi.org/10.1002/pssb.200303855

Cortes, E. et al. (2010). Optical properties of nanostructured metamaterials. Physica Status Solidi (b), 247(8): 2102-7. https://doi.org/10.1002/pssb.200983941

Datta, S., C. T. Chan, K. M. Ho y C. M. Soukoulis. (1993). effective dielectric constant of periodic composite structures. Physical Review B, 48(20): 14936-43, noviembre 15. http://dx.doi.org/10.1103/PhysRevB.48.14936

Decker, M., M. W. Klein, M. Wegener y S. Linden. (2007). Circular dichroism of planar chiral magnetic metamaterials. Optics Letters, 32(7): 856-58, abril 1. http://dx.doi.org/10.1364/OL.32.000856

Haydock, R. (1980). The recursive solution of the Schrödinger equation. Computer Physics Communications, 20(1): 11-16, septiembre 1. http://dx.doi.org/10.1016/0010-4655(80)90101-0

Keller, J. B. (1963). Conductivity of a medium containing a dense array of perfectly conducting spheres or cylinders or nonconducting cylinders. Journal of Applied Physics, 34(4): 991-93, abril 1. http://dx.doi.org/10.1063/1.1729580

Keller, J. B. (1964). A theorem on the conductivity of a composite medium. Journal of Mathematical Physics, 5(4): 548-549.

Klein, Matthias W., Martin Wegener, Nils Feth and Stefan Linden. (2007). OSA Experiments on second- and third-harmonic generation from magnetic metamaterials. Optics Express, 15(8): 5238-5247. OSA | The Optical Society. (Consultado septiembre 2, 2019).

https://www.osapublishing.org/oe/fulltext.cfm?uri=oe-15-8-5238\&id=132271

Krasnok, A., Mykhailo Tymchenko y Andrea Alù. (2018). Nonlinear metasurfaces: a paradigm shift in nonlinear optics. Materials Today, 21(1): 8-21, enero 1. http://dx.doi.org/10.1016/j.mattod.2017.06.007

Krokhin, A., P. Halevi y J. Arriaga. (2002). Long-wavelength limit (homogenization) for two-dimensional photonic crystals. Physical Review B, 65(11): 115208, marzo 7. http://dx.doi.org/10.1103/PhysRevB.65.115208 
Linden S., Enkrich C., Wegener M., Zhou J., Koschny T., Soukoulis C. M. (2004). Magnetic response of metamaterials at 100 terahertz. Science, 306(5700): 1351-53, noviembre 19, Nueva York, N.Y. http://dx.doi.org/10.1126/science.1105371

Liu, Zhaowei, Lee H., Xiong Y., Sun C., Zhang X. (2007). Farfield optical hyperlens magnifying sub-diffraction-limited objects. Science, 315(5819): 1686, marzo 23, Nueva York, N. Y. http://dx.doi.org/10.1126/science.1137368

Markel, Vadim A. (2016). Introduction to the Maxwell Garnett approximation: tutorial. Journal of the Optical Society of America A, 33(7): 1244, julio 1. http://dx.doi.org/10.1364/JOSAA.33.001244

McPhedran, R. C. y G. W. Milton. (1981). Bounds and exact theories for the transport properties of inhomogeneous media. Applied Physics A, 26(4): 207-20, diciembre 1. http://dx.doi.org/10.1007/BF00617840

Mendoza, Bernardo S. y W. Luis Mochán. (2016). Tailored optical polarization in nano structured metamaterials, Physical Review B, 94(19), noviembre 21. http://dx.doi.org/10.1103/PhysRevB.94.195137

Mendoza, Bernardo S. y W. Luis Mochán. (2012). Birefringent nanostructured composite materials. Physical Review B, 85(12): 125418, marzo 14. http://dx.doi.org/10.1103/PhysRevB.85.125418

Meza, Ulises R. Bernardo S. Mendoza y W. Luis Mochán. (2019). Second-harmonic generation in nanostructured metamaterials. Physical Review B, 99(12): 125408, marzo 6. http://dx.doi.org/10.1103/PhysRevB.99.125408

Milton, G. W. (1981). Bounds on the complex permittivity of a two-component composite material. Journal of Applied Physics, 52(8): 5286-93, agosto 1. http://dx.doi.org/10.1063/1.329385

Mochán, W. Luis y Rubén G. Barrera. (1985). Electromagnetic response of systems with spatial fluctuations. I. General formalism. Physical Review B, 32(8): 49844988, octubre 15. http://dx.doi.org/10.1103/PhysRevB.32.4984

Mochán, W. Luis y Rubén G. Barrera. (1985). Electromagnetic response of systems with spatial fluctuations. II. Applications. Physical Review B, 32(8): 4989-5001, octubre 15. http://dx.doi.org/10.1103/PhysRevB.32.4989

Mochán, W. Luis, Bernardo S. Mendoza y Irina Solís. (2014). Second harmonic generation in nanostructured metamaterials. En Latin America Optics and Photonics Conference. Cancún: OSA. LM2C.2. http://dx.doi.org/10.1364/LAOP.2014.LM2C.2

Mochán, W. Luis, Guillermo P. Ortiz y Bernardo S. Mendoza. (2010). Efficient homogenization procedure for the calculation of optical properties of 3D nanostructured composites. Optics Express, 18(21): 22119, octubre 11. http://dx.doi.org/10.1364/OE.18.022119

Nevard, J. y Joseph B. Keller. (1985). Reciprocal relations for effective conductivities of anisotropic media. Journal of Mathematical Physics, 26(11): 2761-65, noviembre 1. http://dx.doi.org/10.1063/1.526697

Ortiz, Guillermo P. et al. (2009). Effective optical response of metamaterials. Physical Review B, 79(24): 245132, junio 26. 
http://dx.doi.org/10.1103/PhysRevB.79.245132

PDL: Minuit -a PDL Interface to the Minuit librarymetacpan.org. (Consultado septiembre 2, 2019). https://metacpan.org/pod/PDL::Minuit

Pendry, J. B. (2000). Negative refraction makes a perfect lens. Physical Review Letters, 85(18): 3966-69, octubre 30.

http://dx.doi.org/10.1103/PhysRevLett.85.3966

Plum, E. et al. (2007). Giant optical gyrotropy due to electromagnetic coupling. Applied Physics Letters, 90(22): 223113, mayo 28.

http://dx.doi.org/10.1063/1.2745203

Popov, A. K. y V. M. Shalaev. (2006). Negative-index metamaterials: second-harmonic generation, manley-rowe relations and parametric amplification. Applied Physics B, 84(1): 131-37, julio 1. http://dx.doi.org/10.1007/s00340-006-2167-4

Schaich, W. L. y A. Liebsch. (1988). Nonretarded hydrodynamic-model calculation of second-harmonic generation at a metal surface. Physical Review B, 37(11): 6187-92, abril 15. http://dx.doi.org/10.1103/PhysRevB.37.6187

Sergio G. Rodrigo, V. Laliena y L. Martín-Moreno. (2015). Second-harmonic generation from metallic arrays of rectangular holes. Journal of the Optical Society of America B, 32(1): 15, enero 1.

Smith, David R. et al. (2000). Composite medium with simultaneously negative permeability and permittivity. Physical Review Letters, 84(18): 4184-87, mayo 1. http://dx.doi.org/10.1103/PhysRevLett.84.4184

Smith, David R. y John B. Pendry. (2006). Homogenization of metamaterials by field averaging (invited paper). Journal of the Optical Society of America B, 23(3): 391, marzo 1. http://dx.doi.org/10.1364/JOSAB.23.000391

Sözüer, H. S., J. W. Haus y R. Inguva. (1992). Photonic bands: Convergence problems with the plane-wave method. Physical Review B, 45(24): 13962-72, junio 15. http://dx.doi.org/10.1103/PhysRevB.45.13962

Tao, Ruibao, Zhe Chen y Ping Sheng. (1990). First-principles Fourier approach for the calculation of the effective dielectric constant of periodic composites. Phys. Rev. B, 41(4): 2417-20, febrero 1. http://dx.doi.org/10.1103/PhysRevB.41.2417

Veselago, Viktor G. (1968). The electrodynamics of substances with simultaneously negative values of and $\mu$. Soviet Physics Uspekhi, 10(4): 509. http://dx.doi.org/10.1070/PU1968v010n04ABEH003699 